\title{
Mit Spiritualität Lebensräume für Kinder und Jugendliche öffnen
}

\section{Christina Aus der Au}

PD Dr. theol., Mitglied der Redaktion Ethik

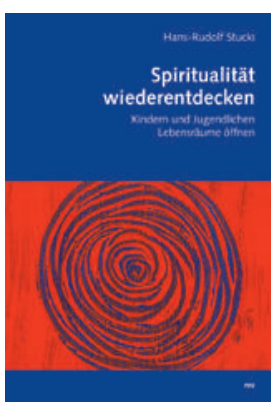

\section{Hans-Rudolf Stucki}

Spiritualität wiederentdecken

Kindern und Jugendlichen

Lebensräume öffnen.

Luzern: rex verlag; 2014.

208 Seiten. 24.80 CHF.

ISBN 978-3-7252-0963-7
Wenn ein Psychiater ein Buch schreibt, in dem der Begriff «Spiritualität» im Titel vorkommt, dann beginnen Theologinnen und Theologen meist sehr skeptisch mit der Lektüre - wenn sie denn das Buch überhaupt zur Hand nehmen. Der Begriff meint oft genug alles und nichts, und das möglichst nicht zu christlich.

Aber Hans-Rudolf Stucki ist nicht nur Arzt mit einer Praxis für Kinder- und Jugendpsychiatrie und Psychotherapie, sondern auch Theologe. Und er definiert Spiritualität schon im ersten Kapitel als «den Bezug zu etwas, was der Mensch hier auf Erden als ihm übergeordnet erfährt, beispielsweise zu etwas Grösserem, Ganzheitlichem», wobei es sich aber «um eine Leben fördernde Grösse handeln» muss.

Diese sehr weitgefasste, aber trotzdem konkret vorgestellte Spiritualität versucht Stucki in der Entwicklung von Kindern und Jugendlichen nachzuverfolgen, und er macht dies an drei Kriterien fest: Spiritualität zeigt sich in existenziellen Fragen, in einem Bezogensein auf eine grössere Wirklichkeit und in einer Selbsttranszendenz bzw. Selbstvergessenheit, in der man sich dieser Wirklichkeit nähert. Stucki macht keinen Hehl daraus, dass er das Christentum als einen in unserem Kulturkreis naheliegenden spirituellen Weg sieht, aber letztlich ist für ihn diese Spiritualität in jeder Religion zu finden. Dies macht es auch kirchendistanzierteren Leserinnen und Lesern leicht, Zugang zu diesem Buch zu finden.

Stucki illustriert seine Thesen mit vielen Beispielen aus der Praxis, die viele Mütter und Väter immer wieder an eigene Erlebnisse mit ihren Kindern erinnern mögen. Wenn er dann Anzeichen von Spiritualität schon bei Zweijährigen findet, so kann man natürlich solche Momente von Selbstvergessenheit oder grossem Staunen auch anders deuten. Stucki versteht die frühkindliche Vorstellung von einem grösseren $\mathrm{Zu}$ sammenhang auch als Teil des Entdeckens der wirklichen Welt, und so ist für ihn Spiritualität und Wissen kein Gegensatz, sondern geeint im Schritt vom Ich zum Anderen.

Hilfreich ist Stuckis Gedanke, dass weniger die Spiritualität selber sich entwickelt, sondern vielmehr deren Ausdruck als religiöse Sozialisierung. Stucki appelliert an die Eltern, diese Sozialisierung selbstkritisch zu reflektieren und - sei es nun allgemein ethisch oder religiös - aktiv zu unterstützen. Sie eröffnen so den Kindern und Jugendlichen einen Deutungshorizont, in dem diese sich und ihr Verhältnis zu den anderen einbetten können.

\section{Stucki illustriert seine Thesen mit vielen Beispielen aus der Praxis.}

Es ist deswegen nachvollziehbar, dass Stucki Spiritualität und den bewussten Umgang damit als wichtige Ressource betrachtet, auch wenn ich selber dann die gesundheitsfördernde Rolle von Religion wesentlich kritischer sehe.

Dass aber der in der Entwicklung des Kindes angelegte Bewegung "weg vom Ich" eine spirituelle und heilsame Dimension innewohnt, die in vielen Religionen begegnet und zu «Lebenswerten und zu Lebenswertem» führt, das zeichnet Stucki praxisnah und differenziert nach. Es ist eine Freude, eine so behutsame Studie zu lesen, die - in der Fülle erschreckender und desillusionierender Nachrichten über Religion - gerade deren Weite und Lebensoffenheit betont. Spiritualität, auch und gerade in ihrer christlichen Ausdrucksform, eröffnet Lebensräume für Kinder und Jugendliche, die auch die Welt der Erwachsenen weiter werden lassen. Stuckis Buch macht nachdenklich und motiviert Eltern und Lehrer/innen, ihre Kinder auch in dieser Beziehung ernst zu nehmen und zu fördern. 